\title{
Polycystic ovary syndrome impact on women's quality of life: pilot study.
}

\author{
Valentina Petkova $^{1 *}$, Maria Kamusheva ${ }^{1}$, Manoela Manova ${ }^{1}$, Alexandra Savova ${ }^{1}, K_{\text {Kalina Andreevska }}{ }^{2}$ \\ ${ }^{1}$ Department of Social Pharmacy, Faculty of Pharmacy, Medical University, Sofia, Bulgaria \\ ${ }^{2}$ Department of Pharmaceutical Science, Faculty of Pharmacy, Medical University, Plovdiv, Bulgaria
}

\begin{abstract}
Purpose: The aim of this study is to assess the impact of Polycystic Ovary Syndrome (PCOS) on Bulgarian patients' QoL through a Polycystic Ovary Syndrome Questionnaire (PCOSQ).

Methods: A pilot survey was conducted in a sample of individuals aged 15 to 35 ( $n=24)$ from Sofia, Bulgaria. Data were collected via PCOSQ. The PCOSQ scale was translated into Bulgarian and standardized by forward translation, backward translation, and a pre-test.

Results: The mean age of the sample was $25.6 \pm 6.4$ (range 15-35). Results from the study found that women with PCOS had a lower appraisal of their appearance because of their weight. The lowest and the highest results were obtained for their mental and emotional status (q.18-1.92 and q.7-4.42). As a factor group-the lowest results were obtained for the questions concerning their overweight-all the 5 questions were in the range of $\mathbf{3 . 2 5 - 3 . 5 8}$. The highest result (-4.42) was reported for in factor group "emotions".

Conclusion: This pilot study found that the mean level of QoL is 3.7 on a scale of 1 to 7 . The main problematic question associated with the disease is women's overweight. The large variance in the levels of emotions of study subjects shows the need to explore this issue further in a larger cohort of patients. It can be concluded that the management of PCOS is urgently needed and the psychological health of these patients must be monitored regularly.
\end{abstract}

Keywords: PCOS, PCOSQ, QoL, Bulgaria.

Accepted on June 29, 2018

\section{Introduction}

Polycystic ovary syndrome was first described by Stein et al. in 1935 and according to them; the symptoms included amenorrhoea and hirsutism in obese women suffering from infertility [1]. Polycystic ovarian syndrome (PCOS) is one of the most widespread endocrine disorders in women of the reproductive age of 12-45 y [2,3]. It is a common disorder of chronically abnormal ovarian function and hyperandrogenism. According to the National Institutes of Health in 1990, there are three main characteristics of PCOS: oligomenorrhoea, hyperandrogenism, and the absence of other endocrine disorders [4]. The presence of polycystic ovaries, as shown by ultrasonography, was not included in the definition but this feature is mandatory. Patients with PCOS complain of infertility, menstrual disturbance, or hirsutism, with or without acne.

At present, there are two main definitions for PCOS, which are also the topic of intense debate. The 1990 National Institutes of Health (NIH) criteria require the presence of chronic anovulation plus clinical or biochemical signs of hyperandrogenism, whereas the 2003 Rotterdam criteria require the presence of two or more of the following: chronic anovulation, clinical or biochemical signs of hyperandrogenism, and polycystic ovaries [5].

Fortunately, in 2003 at a joint consensus meeting between the American Society of Reproductive Medicine and the European Society of Human Reproduction and Embryology, a unifying definition of the PCOS was proposed, encompassing a description of the morphology of the polycystic ovary, ovulatory disorder and hyperandrogenism. The prevalence of polycystic ovary syndrome, as defined by the 1990 National Institutes of Health (NIH) criteria, in unselected populations of women of reproductive age is between $6.5 \%$ and $8 \%$ [6]. According to another survey, it affects 5\%-10\% of women of reproductive age [7]. In general worldwide, PCOS affects up to $6 \%-10 \%$ of this population [8].

The prevalence of PCOS varies depending on which criteria are used to make the diagnosis, but is as high as $15 \%-20 \%$ when the European Society for Human Reproduction and Embryology/American Society for Reproductive Medicine criteria are used [9]. The prevalence of PCOS has been determined among women in different countries but with different findings. A study conducted in China demonstrated a $2.2 \%$ prevalence of PCOS, while a study conducted in Mexico reported a $6 \%$ prevalence of PCOS among women of 
reproductive age according to National Institute of Health criteria $[10,11]$. In addition, a prospective study conducted in unselected Caucasian women from Spain recorded a $6.3 \%$ prevalence of PCOS [12].

PCOS is one of the most common endocrine disorders in women of reproductive age that has substantial psychological, social, and economic consequences. Decreased quality of life characterized by symptoms of depression, decreased sexual satisfaction, and problems with female self-esteem and selfimage have been reported [13,14]. There are many questionnaires for measuring quality of life (QoL), but the one specifically designed to measure the range of health-related problems experienced by women with PCOS is a health-related quality of life questionnaire (PCOSQ) for women with PCOS [15]. It examines disease-related decreases in QoL in PCOS women.

After performing a detailed document survey on the effects of PCOS on Eastern European women, and particularly Bulgarian women, we did not observe any consistent findings. Therefore, the aim of this study is to assess the impact of PCOS on Bulgarian patients' QoL through PCOSQ.

\section{Methods}

A pilot survey was conducted in a sample of individuals aged 15 to $35(n=24)$ from Sofia, Bulgaria who were diagnosed with PCOS. They were diagnosed according to the European Society of Human Reproduction and Embryology/American Society for Reproductive Medicine criteria. Patients were recruited from pharmacies in Sofia, based on referrals from pharmacists and their disease dossiers. All recruited women were required to be otherwise healthy. Women were excluded from the study if they had another major illness that substantially influenced their quality of life or another cause of androgen excess, e.g. congenital adrenal hyperplasia. The Ethics Committee of the Medical University of Sofia approved the study protocol. All participants provided written informed consent before entering the study, and all questionnaires were made anonymous before evaluation.

Data were collected via the Polycysitc Ovary Syndrome Questionnaire (PCOSQ). The PCOSQ scale was translated into Bulgarian and standardized by forward translation, backward translation, and a pre-test. (WHO Process of translation and adaptation of instruments, [16]). The PCOSQ consists of five domains, each relating to a particular symptom of PCOS, including body hair, emotions, overweight, menstrual problems and infertility. Each question on the PCOSQ is associated with a 7-point scale in which 7 represents optimal function and 1 the poorest function.

The data were processed using the statistical package SPSS 20.0 .

\section{Results}

Of the 24 women who agreed to take part in the study, 24 returned questionnaires $(100 \%)$. The mean age of the sample was $25.6 \pm 6.4$ (range 15-35). The mean duration of the disease is $39.8 \pm 30.2$ months (range 6-96). The mean BMI is $29.8 \pm$ 4.8 and $16.7 \%(n=4)$ were clinically obese $(\mathrm{BMI} \geq 30)$ (Table 1).

Table 1. Socio-demographic characteristics in women with PCOS.

\begin{tabular}{ll}
\hline Characteristics & $\mathbf{n = 2 4}$ \\
\hline Age & $25.6 \pm 6.4$ \\
\hline Duration of disease (months) & $39.8 \pm 30.2$ \\
\hline BMI & $29.8 \pm 4.8$ \\
\hline
\end{tabular}

The 26 questions of PCOSQ were grouped into 5 main domain topics: body hair, emotions, overweight, menstrual problems and infertility and were assessed by a 7-point scale (1-poor/7no problem). Results from the study found that women with PCOS had more negative assessment of their appearance because of their weight. High levels of anxiety and depression due to worrying and self-consciousness due to their disease were reflected in a lower PCOSQ score. The lowest and highest results were obtained for questions concerning their emotional status (q.18-1.92 and q.7-4.42). As a factor group, the lowest results were obtained for the questions concerning their overweight; all the five questions are in the range 3.25-3.58. The highest result (4.42) was documented for the factor group of emotions (Table 2).

Table 2. Survey results-impact score of every question.

\begin{tabular}{|c|c|c|}
\hline Factor & Question no. & Impact score \\
\hline \multirow{4}{*}{ Body hair } & 1 & 3.33 \\
\hline & 9 & 4.08 \\
\hline & 15 & 3.83 \\
\hline & 26 & 3.75 \\
\hline \multirow{10}{*}{ Emotions } & 2 & 3.67 \\
\hline & 4 & 3.91 \\
\hline & 6 & 4.25 \\
\hline & 7 & 4.42 \\
\hline & 11 & 3.5 \\
\hline & 14 & 3.92 \\
\hline & 16 & 4.08 \\
\hline & 17 & 2.92 \\
\hline & 18 & 1.92 \\
\hline & 23 & 3.92 \\
\hline \multirow{4}{*}{ Overweight } & 3 & 3.33 \\
\hline & 10 & 3.58 \\
\hline & 12 & 3.25 \\
\hline & 22 & 3.42 \\
\hline
\end{tabular}




\begin{tabular}{lll}
\cline { 2 - 3 } 24 & 3.5 \\
\hline \multirow{3}{*}{ Menstrual problems } & 8 & 3.42 \\
\cline { 2 - 3 } & 19 & 4.25 \\
\cline { 2 - 3 } & 20 & 4.17 \\
\hline \multirow{3}{*}{ Infertility } & 21 & 3.91 \\
\hline & 13 & 4.08 \\
\hline & 25 & 3.67 \\
\hline
\end{tabular}

The impact score of every factor shows that all the 5 factors are in the same range-between 3.42 and 3.94. The lowest score was for emotional status of the patients, while the highest was reflected in their concern about menstrual disturbances due to PCOS (Table 3). Patients with PCOS reported good quality of life $(\mathrm{QoL})$ "a good bit of the time" and "some of the time".

Table 3. Survey results-impact score of every factor.

\begin{tabular}{ll}
\hline Factor & Mean impact score \\
\hline Body hair & 3.75 \\
\hline Emotions & 3.65 \\
\hline Overweight & 3.42 \\
\hline Menstrual problems & 3.94 \\
\hline Infertility & 3.75 \\
\hline
\end{tabular}

\section{Discussion}

The mean factor scores for the PCOSQ reflect the negative impact PCOS can have upon the quality of life of women with this disease. Weight and emotions appeared to be the most significant aspects of the illness according to this cohort. These results confirm the picture that shows previous studies in the region $[3,17,18]$. The finding that weight caused the most negative impact on quality of life has implications for the management of the condition; furthermore, some studies have shown that $50 \%$ of women with PCOS suffer from obesity or are overweight, and it was concluded that the best method to manage this problem remains unclear [19].

Quality of life is increasingly becoming an outcome measure in the long-term management of chronic conditions that demand coping strategies. This is due to the fact that the clinical results do not assess the physical, emotional and social effects' of the disease on those living with the condition. The social predicaments and developmental tasks that all adolescents face may mediate a patient's ability to cope with PCOS [20].

The small size of the respondents was a limitation of this study. Nonetheless, it serves to highlight some important trends. Future studies are needed to compare patients from different areas of Bulgaria and to assess the effects of standard of living on the QoL of these patients as Sofia is the capital of Bulgaria and has the highest cost-of-living allowance.

\section{Conclusion}

PCOSQ is a good tool for measuring the QoL of women with PCOS in Bulgaria. The findings of this pilot study indicate that the mean level of QoL in Bulgaria is 3.7\% for women with PCOS. The main problematic question and topic associated with the disease is women's overweight. The large variance in the levels of emotions shows the need to explore this issue further in a larger cohort of patients. But overall, it can be concluded that better understanding for the management of PCOS is urgently needed and the psychological health of these patients must be monitored and attended to regularly.

\section{References}

1. Stein I, Leventhal M. Amenorrhea associated with bilateral polycystic ovaries. Am J Obstet Gynecol 1935; 29: 181-191.

2. Kabel A, Alghubayshi A, Moharm F. The impact of polycystic ovarian syndrome, a potential risk factor to endometrial cancer, on the quality of sleep. J Cancer Res Treatment 2016.

3. Teede H, Deeks A, Moran L. Polycystic ovary syndrome: a complex condition with psychological, reproductive and metabolic manifestations that impacts on health across the lifespan. BMC Med 2010; 8: 41.

4. Zawadski J, Dunaif A. Diagnostic criteria for polycystic ovary syndrome: towards a rational approach. Polycystic Ovary Syndrome. Blackwell Scientific Publications, Boston 1992; 377-384.

5. Rotterdam ESHRE/ASRM-Sponsored PCOS Consensus Workshop Group. Revised 2003 consensus on diagnostic criteria and long-term health risks related to polycystic ovary syndrome. Fertil Steril 2004; 81: 19-25.

6. Knochenhauer E, Key T, Kahsar-Miller M, Waggoner W, Boots L, Azziz R. Prevalence of the polycystic ovary syndrome in unselected black and white women of the South-Eastern United States: a prospective study. J Clin Endocrinol Metab 1998; 83: 3078-3082.

7. Azziz R, Woods K, Reyna R, Key T, Knochenhauer E, Yildiz B. The prevalence and features of the polycystic ovary syndrome in an unselected population. J Clin Endocrinol Metab 2004; 89: 2745-2749.

8. March W, Moore V, Willson K, Phillips D, Norman R, Davies M. The prevalence of polycystic ovary syndrome in a community sample assessed under contrasting diagnostic criteria. Hum Reprod 2010; 25: 544-551.

9. Sirmans S, Pate K. Epidemiology, diagnosis, and management of polycystic ovary syndrome. Clin Epidemiol 2013; 6: 1-13.

10. Chen X, Yang D, Mo Y, Li L, Chen Y, Huang Y. Prevalence of polycystic ovary syndrome in unselected women from southern China. Eur J Obstet Gynecol Reprod Biol 2008; 139: 59-64.

11. Moran C, Tena G, Moran S, Ruiz P, Reyna R, Duque X. Prevalence of polycystic ovary syndrome and related 
disorders in Mexican women. Gynecol Obstet Invest 2010; 69: 274-280.

12. Asunción M, Calvo R, San Millán J, Sancho J, Avila S, Escobar-Morreale H. A prospective study of the prevalence of the polycystic ovary syndrome in unselected Caucasian women from Spain. J Clin Endocrinol Metab 2000; 85: 2434-2438.

13. Hahn S, Janssen O, Tan S, Pleger K, Mann K, Schedlowski M. Clinical and psychological correlates of quality-of-life in polycystic ovary syndrome. Eur J Endocrinol 2005; 153: 853-860.

14. Hahn S, Quadbeck B, Elsenbruch S, Gartner R, Finke R, Mann K. Metformin, an efficacious drug in the treatment of polycystic ovary syndrome. Deutsche Medizinische Wochenschrif 2004; 129: 1059-1064.

15. Cronin L, Guyatt G, Griffith L, Wong E, Azziz R, Futterweit W. Development of a health-related quality-oflife questionnaire (PCOSQ) for women with polycystic ovary syndrome (PCOS). J Clin Endocrinol Metab 1998; 83: 1976-1987.

16. World Health Organisation. Process of translation and adaptation of instruments. WHO 2017

17. Diamanti-Kandarakis E, Kouli C, Bergiele A, Filandra F, Tsianateli T, Spina G. A survey of the polycystic ovary syndrome in the Greek island of Lesbos: hormonal and metabolic profile. J Clin Endocrinol Metab 1999; 84: 4006-4011.

18. Schmid J, Kirchengast S, Binstofer EV, Huber J. Infertility caused by PCOS - health-related quality of life among Austrian and Moslem immigrant women in Austria Hum Reprod 2004; 19: 2251-2257.

19. Alvarez-Blasco F, Botella-Carretero J, San Millan J, Escobar-Morreale H. Prevalence and characteristics of the polycystic ovary syndrome in overweight and obese women. Arch Intern Med 2006; 166: 2081-2086.

20. Trent ME, Rich M, Austin SB, Gordon CM. Quality of life in adolescent girls with polycystic ovary syndrome. Arch Pediatr Adolesc Med 2002; 156: 556-560.

\section{*Correspondence to}

Valentina Petkova

Department of Social Pharmacy

Faculty of Pharmacy

Medical University

Sofia

Bulgaria 